\title{
Large-basis shell model studies of light nuclei with a multi-valued $G$-matrix effective interaction
}

\author{
D. C. Zheng and B. R. Barrett \\ Department of Physics, University of Arizona, Tucson, Arizona 85721 \\ J. P. Vary \\ Department of Physics and Astronomy, Iowa State University, Ames, Iowa 50011 \\ W. C. Haxton and C.-L. Song \\ Institute for Nuclear Theory and Department of Physics, University of Washington, Seattle, Washington 98195
}

(June 13, 2021)

\begin{abstract}
Large-basis shell model studies of low-lying excitations in light nuclei from ${ }^{4} \mathrm{He}$ to ${ }^{7} \mathrm{Li}$ have been performed with a multivalued $G$-matrix effective interaction, as recently suggested by Haxton et al.. Calculations were performed relative to the vacuum ("no core") using very large, separable model spaces containing all excitations with unperturbed energies up to $8 \hbar \Omega$. Using $G$ matrices derived from a new Nijmegen potential, we achieve a very satisfactory description of these excitations.
\end{abstract}

21.60.Cs, 21.10.Ky, 27.10.+h

\section{INTRODUCTION}

The nuclear Hamiltonian

$$
H=\frac{1}{2} \sum_{i \neq j}^{A}\left(T_{i j}+V_{i j}\right),
$$

where $T_{i j}$ is the relative kinetic energy operator and $V_{i j}$ the nucleon-nucleon $(N N)$ interaction, is often treated in the nuclear shell model by introducing the one-body harmonic oscillator (HO) Hamiltonian

$$
H_{0}=\sum_{i=1}^{A} h_{i}=\sum_{i=1}^{A}\left(\frac{\boldsymbol{p}_{i}^{2}}{2 M}+\frac{1}{2} M \Omega^{2} \boldsymbol{r}_{i}^{2}\right)
$$

to classify the many-body states: Slater determinants are formed from the products of these single-particle wave functions. These many-body basis states can be labeled according to the number of oscillator quanta they contain, $N_{\text {sum }}=\sum_{i=1}^{A} N_{i}$, or, equivalently, the unperturbed energies

$$
\sum_{i=1}^{A}\left(N_{i}+\frac{3}{2}\right) \hbar \Omega
$$

where $N_{i}$ is the number of oscillator quanta $\left(2 n_{i}+l_{i}\right)$ of the $i$ th single-particle state. Conventionally the labeling is relative to the minimum energy Slater determinant(s), so that the basis states are partitioned into $0 \hbar \Omega, 1 \hbar \Omega$, $2 \hbar \Omega$, etc., configurations.

Early shell model calculations were generally restricted to a single shell, such as the $0 p$ or $1 s 0 d$ shells, and thus involved only $0 \hbar \Omega$ valence nucleon configurations. An effective interaction is then introduced to account for the effects of excluded configurations, including very high energy excitations associated with the hard core in the $N N$ interaction. The lowest order approximation to this effective interaction is the two-body $G$ matrix, which links two-particle states within the model space by a ladder series for scattering in the excluded space. The resulting interaction $V^{\mathrm{eff}}(a b ; c d)$ is a function of the valence-shell single particle states $c, d$ and $a, b$ that label the starting and ending states of the ladder, respectively.

In recent years shell model calculations involving two or more major shells have been frequently performed. A full multi- $\hbar \Omega$ basis is one that includes all many-body configurations, such that $N_{\text {sum }} \leq N_{\max }$ for some $N_{\max }$. For example, a calculation of the positive-parity states in ${ }^{16} \mathrm{O}$ might include all $(0+2) \hbar \Omega$ or $(0+2+4) \hbar \Omega$ many-body configurations, relative to the closed core (fully occupied $0 s$ and $0 p$ shells). Because of the importance of the nuclear mean field, such a truncation provides a reasonable starting point for describing the "long-wavelength" properties of nuclei. A $(0+2+4) \hbar \Omega$ calculation (for which $N_{\max }=16$ ) of ${ }^{16} \mathrm{O}$ yields a reasonable description of low-lying excitations, including effects associated with highly deformed excited states [1].

Such full multi- $\hbar \Omega$ bases have other appealing properties. If $\mathrm{HO}$ single-particle states are employed, the model space wave functions can be decomposed so that the relative degrees of freedom are separated from a pure oscillator state center-of-mass component. Thus the overcompleteness of the Slater determinants (which depend on $3 A$ coordinates, while intrinsic wave functions depend on $3(A-1))$ can be cured by retaining only those linear combinations which keep the center of mass in the $0 s$ state.

A second property has to do with technical difficulties in evaluating the effective interaction. If, in addition to defining the basis states, $H_{0}$ of Eq. (2) plays the role of the unperturbed Hamiltonian, then the unperturbed 
energies of configurations in the excluded space always exceed those in the model space. In the case of parityconserving (parity-nonconserving) interactions, the minimum gap is $2 \hbar \Omega(1 \hbar \Omega)$. This contrasts sharply with other choices for the model space. For example, a partial $1 \hbar \Omega$ calculation of the negative parity states in ${ }^{12} \mathrm{C}$ in which the valence nucleons are restricted to the $0 p$ and $1 s 0 d$ shells leads to intermediate states in the core-polarization process (bubble diagram) with vanishing energy denominators: particle-hole excitations in the excluded space of the form $0 p(0 s)^{-1}$ have the same energy, $1 \hbar \Omega$, as those in the model space. While one might attempt to cure this problem by introducing a spin-orbit interaction in $H_{0}$ to break the degeneracy, this tends to produce small energy denominators of somewhat random sign, leading to serious convergence problems. Thus one sees that the gaps characterizing complete multi- $\hbar \Omega$ bases are quite attractive. As discussed in Ref. [2], this nice feature can be perserved order by order in calculations of the full $V^{\mathrm{eff}}$, provided a suitable perturbation scheme for $V^{\mathrm{eff}}$ is employed.

Investigators doing large-basis shell model calculations in multi- $\hbar \Omega$ spaces have consistently chosen effective twobody interactions of the form $V^{\text {eff }}(a b ; c d)$, just as in traditional $0 \hbar \Omega$ calculations. The appropriate effective interaction [2] in such spaces must carry an additional index $N_{\text {sum }}^{\text {spectators }} V^{\text {eff }}\left(a b ; c d ; N_{\text {sum }}^{\text {spectators }}\right)$, where $N_{\text {sum }}^{\text {spectators }}$ labels the total oscillator quanta of the "spectator" (i.e., non-interacting) nucleons in the many-body states connected by the matrix element $V^{\mathrm{eff}}(a b ; c d)$. It is given by

$$
N_{\text {sum }}^{\text {spectators }}=N_{\text {sum }}-N_{c}-N_{d}=N_{\text {sum }}^{\prime}-N_{a}-N_{b},
$$

where $N_{\text {sum }}$ and $N_{\text {sum }}^{\prime}$ are the numbers of the total oscillator quanta of the initial and final many-body states, respectively. In the case of traditional $0 \hbar \Omega$ calculations, all basis states are characterized by the same $N_{\text {sum }}^{\text {spectators }}$, so this additional index is unnecessary. But for multi- $\hbar \Omega$ bases, the $N_{\text {sum }}^{\text {spectators }}$ dependence is essential: if modelspace configurations exist with different unperturbed energies, the gaps and interactions coupling these configurations to the excluded space will differ. The appropriate energy denominators in the $G$-matrix ladder sum are not given just by the initial and final two-particle labels, but also depend on the energies of the $A-2$ "spectator" nucleons.

The omission of the $N_{\text {sum }}^{\text {spectators }}$ dependence in recently reported large-space shell-model calculations [3, amounts to neglect of certain many-body processes of the same unperturbed energy as some retained manybody processes. While the effects of these neglected many-body processes are expected to decrease in importance as the number of shells included in the model space increases, our investigation here, which retains them through the $N_{\text {sum }}^{\text {spectators }}$ dependence of the two-body effective interaction, will reveal that these neglected effects are important in present-day calculations.
We shall see in the following calculations that the resulting shifts can be large, amounting to about $5 \mathrm{MeV}$ for diagonal matrix elements. The approximation in presentday multi-shell calculations to neglect the $N_{\text {sum }}^{\text {spectators }}$ dependence can lead to unattractive consequences. One example is the apparent need for unrealistic single particle energies to reduce the splittings between the $0 \hbar \Omega$ and $2 \hbar \Omega$ states, as required by experiment.

In this paper we present the results of multi- $\hbar \Omega$ shell model calculations for ${ }^{4} \mathrm{He},{ }^{5} \mathrm{He},{ }^{6} \mathrm{Li}$, and ${ }^{7} \mathrm{Li}$ in which the two-body effective interaction is evaluated with full $N_{\text {sum }}^{\text {spectators }}$ dependence. As a result, we obtain a lowering of states that are dominated by $1 \hbar \Omega$ and $2 \hbar \Omega$ configurations, relative to $0 \hbar \Omega$ states. This improves the agreement with experiment. The calculation of the $G$ matrix is described in Sec. II. This work is distinguished from our previous studies [3, 3 ] in another important aspect, namely, the extension of the model spaces for light nuclei to include excitations up to $8 \hbar \Omega$. These calculations are "no core," performed relative to vacuum and, of course, include excitations out of the $0 s$ shell. The results are presented in Sec. III, where a comparison with previous calculations is also made. The dependence of the results on the size of the model space is discussed in Sec. IV, and the consequences of neglecting the $N_{\text {sum }}^{\text {spectators }}$ dependence of the $G$ matrix explored. Our conclusions are given in Sec. V.

\section{MULTI-VALUED G-MATRIX EFFECTIVE INTERACTIONS}

Shell model diagonalizations of the Hamiltonian in Eq. (11) are performed within truncated Hilbert spaces containing, hopefully, most of the long-wavelength modes important to describing properties such as nuclear sizes, low-lying excitations and collective modes. The neglected degrees of freedom, e.g., those high-momentum interactions arising from $N N$ interactions at short distances, must be incorporated into the calculation through effective interactions (and effective operators). While in principle an effective interaction exists that will reproduce exact eigenvalues in a model space calculation, in practice it can only be evaluated approximately.

The shell model Hamiltonian we diagonalize is

$$
\begin{aligned}
H_{\mathrm{SM}}= & \frac{1}{2} \sum_{i \neq j}^{A}\left(T_{i j}+V_{i j}^{\mathrm{eff}}\left(N_{\mathrm{sum}}^{\text {spectators }}\right)\right)+V_{\text {Coulomb }} \\
& +\lambda\left(H_{\text {c.m. }}-\frac{3}{2} \hbar \Omega\right)
\end{aligned}
$$

where $T_{i j}=\frac{1}{2 A M}\left(\boldsymbol{p}_{i}-\boldsymbol{p}_{j}\right)^{2}$ and

$$
\frac{1}{2} \sum_{i \neq j}^{A} T_{i j}=\sum_{i=1}^{A} \frac{\boldsymbol{p}_{i}^{2}}{2 M}-T_{\text {c.m. }} .
$$


with $T_{\text {c.m. }}=\frac{1}{2 A M}\left(\sum_{i=1}^{A} \boldsymbol{p}_{i}\right)^{2}$. Note that $H_{\text {c.m. }}=$ $T_{\text {c.m. }}+U_{\text {c.m. }}$ where $U_{\text {c.m. }}=\frac{A M \Omega^{2}}{2}\left(\sum_{i=1}^{A} \boldsymbol{r}_{i}\right)^{2}$. The last term in Eq. (5) is included in order to project out spurious center-of-mass motion: inclusion of this term with a large value of $\lambda$ produces low-lying excitations with the center-of-mass in the $0 s$ state. For this procedure to work properly, the model space must be exactly separable, as is the case for complete multi- $\hbar \Omega$ bases.

The bare Coulomb interaction $V_{\text {Coulomb }}$ is diagonalized only within the model space. For the strong potential, the effective interaction evaluated at the two-body level has the familiar Bruckner $G$-matrix [5] form, but with an important difference in the definition of the Pauli exclusion operator $Q$,

$$
\begin{gathered}
G\left(E_{0}, N_{\text {sum }}^{\text {spectators }}\right)=V_{12}+\tilde{V}_{12} Q\left(N_{\text {sum }}^{\text {spectators }}\right) \\
\times \frac{1}{E_{0}-\left(h_{1}+h_{2}+\tilde{V}_{12}\right)} Q\left(N_{\text {sum }}^{\text {spectators }}\right) \tilde{V}_{12},
\end{gathered}
$$

where $E_{0}$ is the energy of the initial two-body state (i.e., the starting energy), $V_{12}$ is the bare $N N$ potential, $\tilde{V}_{12}=V_{12}-U_{12}, U_{12}=\frac{M \Omega^{2}}{2 A}\left(\mathbf{r}_{1}-\mathbf{r}_{2}\right)^{2}$, and $Q\left(N_{\text {sum }}^{\text {spectators }}\right)$ is the Pauli operator that restricts all intermediate states to lie in the Pauli-allowed, excluded space. In conventional treatments of the Brueckner $G$ matrix, a very similar equation arises, but with a $Q$ that excludes certain intermediate states based only on the single-particle labels of the two particles involved in the scattering. There is no $N_{\text {sum }}^{\text {spectators }}$ dependence in $Q$ for single shell calculations but, as discussed above, this $N_{\text {sum }}^{\text {spectators }}$ dependence arises in multi- $\hbar \Omega$ calculations.

The index $N_{\text {sum }}^{\text {spectators }}$ signifies the role of the full model space many-body configuration in controlling the intermediate two-particle states available for scattering. In a shell model calculation whose model space includes all many-body states with $N_{\text {sum }} \leq N_{\max }$, the allowed intermediate states for the two particles, "1" and "2", scattered by $\tilde{V}_{12}$, are specified by:

$$
N_{1}+N_{2}+N_{\text {sum }}^{\text {spectators }}>N_{\text {max }},
$$

which corresponds to the following Pauli operator:

$Q\left(N_{\text {sum }}^{\text {spectators }}\right)= \begin{cases}0 & \text { if } N_{1}+N_{2} \leq N_{\text {max }}-N_{\text {sum }}^{\text {spectators }} \\ 1 & \text { otherwise. }\end{cases}$

In Fig.1, we depict the various spectator-dependent Pauli operators appropriate for a full $6 \hbar \Omega$ calculation of ${ }^{6} \mathrm{Li}$ $\left(N_{\max }=8\right)$.

The fact that we introduce a spectator dependence to the $G$-matrix raises interesting possibilities for identifying specific Pauli-violating processes. Some two-particle scattering states in the excluded space will place a nucleon in a single-particle state that may be occupied by a spectator nucleon in a given model space wavefunction. One might avoid these Pauli violating processes in a full multi- $\hbar \Omega$ calculation by labeling $Q$ with the full set of quantum numbers on which $G$ operates. This, of course, is impractical. However, for the specific case of these light nuclei and for $N_{\text {sum }}^{\text {spectators }}=0$, we can easily eliminate the Pauli violating processes involving the $0 s$ nucleons by including the "wings" as depicted in Fig. 1. However, we have found that the presence or absence of the wings in the case $N_{\text {sum }}^{\text {spectators }}=0$ results in minor differences in our results due to the large size of the model spaces.

To provide the reader with some measure of the size of the effects associated with $N_{\text {sum }}^{\text {spectators }}$, we give in Table I the matrix elements $\left\langle\left(0 s_{1 / 2} 0 s_{1 / 2}\right)\left|V^{\text {eff }}\right|\left(0 s_{1 / 2} 0 s_{1 / 2}\right)\right\rangle$, $\left\langle\left(0 s_{1 / 2} 0 p_{3 / 2}\right)\left|V^{\mathrm{eff}}\right|\left(0 s_{1 / 2} 0 p_{3 / 2}\right)\right\rangle, \quad$ and $\left\langle\left(0 p_{3 / 2} 0 p_{3 / 2}\right)\left|V^{\text {eff }}\right|\left(0 p_{3 / 2} 0 p_{3 / 2}\right)\right\rangle$, that we evaluated for a full $6 \hbar \Omega$ calculation of the positive-parity states in ${ }^{6} \mathrm{Li}$ (which we will discuss in Sec. [IIC). In this calculation, $N_{\text {max }}=8$ and $N_{\text {sum }}$ can take on four values $(2,4,6,8)$. The Table shows that the values of these diagonal matrix elements can shift by up to $3.3 \mathrm{MeV}$ when $N_{\text {sum }}^{\text {spectators }}$ dependence is properly treated.

This "multi-valuedness" is a bookkeeping complication in shell model studies. However, its inclusion builds in essential physics previously missing from multi- $\hbar \Omega$ calculations. Model-space states of higher unperturbed energy are now more strongly repelled downwards by effects of states in the excluded space which are included in $G$ for the first time. For example, in a $(0+2+4) \hbar \Omega$ calculation of ${ }^{16} \mathrm{O}$, the contribution to $G$ that is second order in $\tilde{V}_{12}$ contributes to shifts in the position of the $0 \hbar \Omega$ configuration only because of potential matrix elements with an unperturbed energy denominator of $6 \hbar \Omega$ or larger. However, the $4 \hbar \Omega$ configurations are shifted by matrix elements with an energy denominator of $2 \hbar \Omega$ or larger. The larger shifts result from a more complete inclusion of intermediate two-particle states scattering via a spectator dependent definition of $Q$, which reduces the size of the $Q=0$ region as $N_{\text {sum }}^{\text {spectators }}$ increases. Since $G$ for a larger $Q=0$ region is less "attractive", numerically, we find that states that are predominantly of $1 \hbar \Omega$ and $2 \hbar \Omega$ character, which were obtained at energies a few $\mathrm{MeV}$ too high in Ref. [⿰], are now lowered relative to states that are predominantly $0 \hbar \Omega$ in character.

The intent of the present study is to illustrate the effects attributable to the multivaluedness of $G$. We therefore follow the treatment in Ref. [4] in other respects, which includes two approximations in the evaluation of Eq. (7). The first is the substitution of $V$ for $\tilde{V}=V-U$. This considerably improves the convergence of the numerical procedures we employ in evaluating $G$, since the growth of the HO $U(r)$ at large $r$ is troublesome. Earlier studies [6] have shown that neglecting $U$ in terms second order or higher in $V$ induces errors in calculated binding energies of a few hundred $\mathrm{keV}$; these errors decrease as the size of the model space increases.

The second is the replacement of the "starting energy" $E_{0}$ in the ladder sum of Eq. (7) by 


$$
\omega_{c d}=\epsilon_{c}+\epsilon_{d}+\Delta
$$

where $\left(\epsilon_{c}+\epsilon_{d}\right)$ is the unperturbed energy of the initial two-body state and $\Delta$ is a parameter whose value is adjusted to yield a reasonable binding energy. This substitution was introduced and explained in Ref. [4]: it is a phenomenological correction for the omission of folded diagrams and higher-order contributions to the effective interaction, those beyond $G$ that involve the multiple scattering of clusters of three or more nucleons. As some of these omitted corrections will shift starting energies from their unperturbed values towards the true eigenvalues, it is not surprising that a phenomenological shift $\Delta$ is necessary to reproduce experimental binding energies when a two-body $G$ matrix is used. However, energy differences are relatively insensitive to the choice of $\Delta$. Such a state-dependent choice for the starting energy leads to a non-Hermitian $G$ matrix. But the non-Hermiticity is found to be small and we obtain a Hermitian effective interaction by symmetrizing the $G$ matrix:

$$
\begin{aligned}
& \left\langle a b\left|V^{\mathrm{eff}}\left(N_{\mathrm{sum}}^{\text {spectators }}\right)\right| c d\right\rangle_{J, T} \\
= & \frac{1}{2}\left[\left\langle a b\left|G\left(\omega_{c d}, N_{\mathrm{sum}}^{\text {spectators }}\right)\right| c d\right\rangle_{J, T}\right. \\
+ & \left.\left\langle a b\left|G\left(\omega_{a b}, N_{\mathrm{sum}}^{\text {spectators }}\right)\right| c d\right\rangle_{J, T}\right] .
\end{aligned}
$$

We employ the method of Barrett et al. [7] to calculate the $G$ matrices. For the bare $N N$ interaction $V_{12}$ in Eq. (7), we use the non-relativistic version of the new Nijmegen potential (NijmII) [8]. The HO basis parameter $\hbar \Omega$ is fixed at $14 \mathrm{MeV}$. Calculations for a different choice of $\hbar \Omega$ are performed for selected cases for the purpose of comparison.

\section{RESULTS AND DISCUSSION}

The shell-model calculations are performed for ${ }^{4} \mathrm{He}$, ${ }^{5} \mathrm{He},{ }^{6} \mathrm{Li}$, and ${ }^{7} \mathrm{Li}$ in large, no-core, model spaces using the Many-Fermion-Dynamics Shell-Model code [9]. We properly evaluate the $G$ matrix for full multi- $\hbar \Omega$ spaces, resulting in a multi-valued two-body effective interaction. The calculated results are presented in Tables I to IV which we discuss below.

\section{A. ${ }^{4} \mathrm{He}$}

For the positive-parity states in ${ }^{4} \mathrm{He}$, we use a 9 -majorshell model space which allows us to include all configurations with $N_{\text {sum }}=N_{1}+N_{2}+N_{3}+N_{4} \leq 8$ [i.e., $\left.N_{\max }=8\right]$. For the negative-parity states, we use a 8-major-shell space and include all configurations with $N_{\text {sum }} \leq N_{\max }=7$. The lowest configuration in this nucleus is $(0 s)^{4}$, which has $N_{\text {sum }}=0$, so we are doing a full $8 \hbar \Omega(7 \hbar \Omega)$ calculation for the positive-parity (negativeparity) states. The calculations involve $\left(N_{\max }+1\right) G$ matrices, corresponding to $\left(N_{\max }+1\right)$ possible values of $N_{\text {sum }}^{\text {spectators }}$ (from 0 to $N_{\max }$ ).

The parameter $\Delta$ in the starting energy is chosen to be $-55 \mathrm{MeV}$, which yields a reasonable binding energy of $26.3 \mathrm{MeV}$. (It should be pointed out that due to the large size of the model space, the $G$ matrix elements are a very smooth function of $\Delta$. The binding energy of ${ }^{4} \mathrm{He}$ increases by less than $1 \mathrm{MeV}$ when $\Delta$ is increased by $10 \mathrm{MeV}$ from $-60 \mathrm{MeV}$ to $-50 \mathrm{MeV}$. See Ref. [4] for a discussion of the sensitivity of the results to $\Delta$.) The calculated results are given in Table II and plotted in Fig.2 along with the experimental data, taken from a recent compilation of Tilley et al. [10] and Ref. [11].

As can be seen from Table II and Fig.2, very good agreement with experiment is obtained for the energy spectrum. In particular, the experimental low-lying negative-parity (" $1 \hbar \Omega$ ") states are reproduced to within 1.2 MeV with a correct level sequence. The first excited $0^{+}$(predominantly " $2 \hbar \Omega$ ") state is obtained at an excitation energy of $21.8 \mathrm{MeV}$, only $1.6 \mathrm{MeV}$ higher than experiment.

The importance of the high-energy configurations can be seen by examining the wave functions. In terms of major-shell configurations, the calculated g.s. wave function can be expressed as

$$
\begin{aligned}
{\left[0_{1}^{+}\right] \simeq } & 70 \%|0 \hbar \Omega\rangle+14 \%|2 \hbar \Omega\rangle+9 \%|4 \hbar \Omega\rangle \\
& +3 \%|6 \hbar \Omega\rangle+4 \%|8 \hbar \Omega\rangle
\end{aligned}
$$

while for the first excited state, we obtain

$$
\begin{aligned}
{\left[0_{2}^{+}\right] \simeq } & 8 \%|0 \hbar \Omega\rangle+61 \%|2 \hbar \Omega\rangle+15 \%|4 \hbar \Omega\rangle \\
& +13 \%|6 \hbar \Omega\rangle+3 \%|8 \hbar \Omega\rangle .
\end{aligned}
$$

As can be expected, the $0_{1}^{+}$state is dominated by the $0 \hbar \Omega$ configuration and the $0_{2}^{+}$state is dominated by the $2 \hbar \Omega$ configuration. However, we see from the above "wave functions" that $\left|0_{1}^{+}\right\rangle$has significant $2 \hbar \Omega$ and $4 \hbar \Omega$ admixtures while $\left|0_{2}^{+}\right\rangle$has significant $4 \hbar \Omega$ and $6 \hbar \Omega$ admixtures. Therefore, for a reasonable description of the $0_{1}^{+}$and $0_{2}^{+}$ states using a $\mathrm{HO}$ basis with $\hbar \Omega=14 \mathrm{MeV}$, one needs to perform a $4 \hbar \Omega$ calculation and a $6 \hbar \Omega$ calculation, respectively. The requirement of a large $\mathrm{HO}$ space for convergence of wave functions dominated by $1 \hbar \Omega$ and $2 \hbar \Omega$ components has been established by Ceuleneer et al. in Ref. 112 where a $10 \hbar \Omega$ calculation was performed for ${ }^{4} \mathrm{He}$ using a modified Sussex 13 interaction. Because the $\mathrm{HO}$ potential is too confining at large distances, high-lying configurations are required to properly describe the shape of the nuclear surface. Alternatively, one may address these same physics issues within the effective Hamiltonian formalism in a $\mathrm{HO}$ space by evaluating the contributions of effective many-body interactions.

The weights of the different major-shell configurations listed in Eq. (12) and Eq. (13) depend on the choice of single-particle basis: they would change if we were to adopt a Hartree-Fock basis or, even, retain $\mathrm{HO}$ wave functions but change the value of the oscillator parameter. One procedure for removing this arbitrariness, for a 
given model space, would be to diagonalize the ground state one-body density matrix, then transform to a new basis given by the eigenvectors. The naive "closed shell" would then be defined by the largest eigenvalues. We will discuss these issues further in Sec. IV.

The major differences in the spectra resulting from the present work and Refs. 3, 3 , appear in the lowering of the excited states due to increased admixtures of higher lying configurations for the reasons mentioned above. For example, the $0_{2}^{+}$state is lowered by $11.9 \mathrm{MeV}$ from its excitation energy in Ref. [3] and by $4.3 \mathrm{MeV}$ from its excitation energy in Ref. [4]. On the other hand, the $0_{1}^{-}$ state is lowered by only $0.8 \mathrm{MeV}$ and $1.3 \mathrm{MeV}$ relative to its excitation energy in Ref. [3] and Ref. [4], respectively.

Since there are a number of differences between the present work and our previous efforts, we will discuss in Sec. IV the dependence of our results on model space size alone with all other ingredients in the calculations held fixed.

\section{B. ${ }^{5} \mathrm{He}$}

Throughout this work, the unperturbed energy of a configuration is measured with respect to that of the lowest configuration (of either parity). For ${ }^{5} \mathrm{He}$, the lowest $(0 \hbar \Omega)$ configuration is $(0 s)^{4}(0 p)^{1}$ with $N_{\text {sum }}=1$. We do a full $6 \hbar \Omega$ calculation $\left(N_{\max }=7\right)$ for the negative-parity states and a full $7 \hbar \Omega$ calculation $\left(N_{\max }=8\right)$ for the positive-parity states. The parameter $\Delta$ in the starting energy is taken to be $-45 \mathrm{MeV}$ for this and other $0 p$-shell nuclei considered in this work. The results are shown in Table III and in Fig.3. The first excited state $\left(1 / 2^{-}\right)$ is obtained at an excitation energy of $2.47 \mathrm{MeV}$. The well-known $3 / 2^{+}$state at $16.75 \mathrm{MeV}$ is reproduced at an energy of $19.06 \mathrm{MeV}$. We have also obtained a number of low-lying " $1 \hbar \Omega$ " positive-parity and " $2 \hbar \Omega$ " negativeparity states that have not yet been identified experimentally. There is a $1 / 2^{+}$state at an excitation energy of only $4.34 \mathrm{MeV}$ and there are two nearly degenerate $3 / 2^{+}$and $5 / 2^{+}$states at about $9.7 \mathrm{MeV}$. The lowest " $2 \hbar \Omega$ " state $\left(3 / 2^{-}\right)$is obtained at an excitation energy of $12.01 \mathrm{MeV}$.

The energy splitting $\Delta E$ between the $1 / 2^{-}$state and the $3 / 2^{-}$state (g.s.) is of particular interest. Analyses of experimental data yielded many different values for $\Delta E$, ranging from $1.4 \mathrm{MeV}$ [14] to more than $5 \mathrm{MeV}$ [15]. In a recent Green's function Monte Carlo (GFMC) calculation [16] where the odd neutron is restricted to be in the $p_{1 / 2}$ or $p_{3 / 2}$ state (not pure HO $0 p$ states), a small splitting of $0.8 \mathrm{MeV}$ is obtained. In our calculations, we notice that $\Delta E$ tends to decrease as we include more $p$ orbitals in the model space. The values for $\Delta E$ obtained in the $0 \hbar \Omega, 2 \hbar \Omega, 4 \hbar \Omega$ and $6 \hbar \Omega$ calculations are $2.81,3.15$, 2.89 and $2.47 \mathrm{MeV}$, respectively. As this series does not appear to have converged, it is quite possible that still larger model spaces would yield a result below the $6 \hbar \Omega$ value of $2.47 \mathrm{MeV}$.
Our predictions of the low-lying positive-parity states agree quite well with other theoretical works [17, 18 where a $1 / 2^{+}$state at about $5-7 \mathrm{MeV}$ is predicted along with two additional states $\left(3 / 2^{+}\right.$and $\left.5 / 2^{+}\right)$at about 12 $14 \mathrm{MeV}$, except that our results are slightly lower. These levels were first obtained by van Hees and Glaudemans 117] in a $(0+1) \hbar \Omega$ shell-model calculation using a phenomenological interaction (obtained by fitting selected nuclear properties) and were later supported by other shell-model calculations using different phenomenological interactions [18]. These states are expected to be broad and cannot be easily identified experimentally. However, they can be seen in an R-matrix analysis of the nucleonalpha phase shifts with a channel radius of $a \sim 5 \mathrm{fm}$ [18], but not with a smaller $a$ of about $3 \mathrm{fm}$ commonly used before. A large channel radius of $5.5 \pm 1.0 \mathrm{fm}$ has been determined from the stripping and pickup reaction data [15.

These low-lying positive-parity states were obtained at higher energies in our previous $3 \hbar \Omega$ (i.e., $N_{\max }=4$ ) single- $G$ calculation [4]. This can be explained by noting that the calculated wave functions of these states contain significant higher-shell configurations. For example, for the $1 / 2^{+}$state, we obtain

$$
\left[1 / 2_{1}^{+}\right]=45 \%|1 \hbar \Omega\rangle+28 \%|3 \hbar \Omega\rangle+18 \%|5 \hbar \Omega\rangle+9 \%|7 \hbar \Omega\rangle .
$$

The calculated wave functions also show that these states can be roughly described as systems with one neutron moving in an $s$ or $d$ (not necessarily $1 s$ or $0 d$ ) orbitals outside a ${ }^{4} \mathrm{He}$ core.

The point made by Eq. (14) and the associated discussion may appear provocative. If we try to interpret all our states as predictions for the locations of resonances, this would indicate the near absence of a shell gap in ${ }^{5} \mathrm{He}$. However, we should always keep in mind that ${ }^{5} \mathrm{He}$ is unbound with respect to neutron emission and that all the states of ${ }^{5} \mathrm{He}$ are experimentally above breakup threshold (i.e. in the continuum). Thus, as we systematically expand our model space we would expect our calculated results to approach a continuous spectrum, though presumably the convergence might be quite slow due to the use of confined $\mathrm{HO}$ wave functions in the shell model expansion. By analyzing transition strength functions we would be able to isolate those states which are truly predicted resonances from the background of continuum states. However, at the present time, our model space is too limited to be able to carry out such an analysis. Nevertheless, we expect that states which are experimentally narrow will be reproduced by our theoretical framework.

The calculated $3 / 2^{+}$state at $19.06 \mathrm{MeV}$ is dominated by the configuration $(0 s)^{3}(0 p)^{2}$, which is basically the ground state of ${ }^{6} \mathrm{Li}$ with a proton removed from the $0 \mathrm{~s}$ orbital. It can therefore be identified as the $16.75 \mathrm{MeV}$ state observed experimentally in nucleon knock-out reactions with a ${ }^{6} \mathrm{Li}$ target [19]. 
The lowest " $2 \hbar \Omega$ " state is calculated at a surprisingly low excitation energy of $12.01 \mathrm{MeV}$ with $\left(J^{\pi}, T\right)=$ $\left(3 / 2^{-}, 1 / 2\right)$. This state was obtained at a much higher energy of $21.5 \mathrm{MeV}$ in a $4 \hbar \Omega$ (i.e., $N_{\max }=5$ ) single- $G$ calculation [4]. The dramatic decrease of the excitation energy is due, again, to the importance of $6 \hbar \Omega$ admixtures, as can be seen from the following decomposition:

$$
\left[3 / 2_{2}^{-}\right]=1 \%|0 \hbar \Omega\rangle+52 \%|2 \hbar \Omega\rangle+28 \%|4 \hbar \Omega\rangle+19 \%|6 \hbar \Omega\rangle .
$$

The fact that the above wave function contains a large $6 \hbar \Omega$ component implies that the energy of this state, now at $12.01 \mathrm{MeV}$, is likely to be further decreased if one is able to do an even larger calculation to include the $8 \hbar \Omega\left(N_{\max }=9\right)$ configuration. The second lowest " $2 \hbar \Omega$ " state is obtained at $15.21 \mathrm{MeV}$ with $\left(J^{\pi}, T\right)=$ $\left(1 / 2^{-}, 1 / 2\right)$. These two " $2 \hbar \Omega$ " states were also obtained by Wolters et al. 20] in a $(0+2) \hbar \Omega$ calculation using an phenomenological effective interaction, but at an even lower energy of about $9 \mathrm{MeV}$ (see, however, Ref. 21] for a comment on this work).

\section{C. ${ }^{6} \mathbf{L i}$}

For this nucleus, we perform a full $6 \hbar \Omega$ calculation $\left(N_{\max }=8\right)$ for the positive-parity states and a full $5 \hbar \Omega$ calculation $\left(N_{\max }=7\right)$ for the negative-parity states. The results are shown in Table IV and in Fig.4. The six low-lying states known experimentally are nicely reproduced except that the $J^{\pi}=2^{+}, T=1$ state at $5.37 \mathrm{MeV}$ and the $J^{\pi}=1^{+} T=1$ state at $5.65 \mathrm{MeV}$ are obtained at excitation energies about $1 \mathrm{MeV}$ too high. The other four " $0 \hbar \Omega$ " states are obtained at excitation energies of 9.94 , $10.74,11.38$ and $12.93 \mathrm{MeV}$. The new results presented here again show some improvement over the previous results [3, 4]. The excitation energies for the first and the second excited states are closer to experiment than those obtained in the GFMC calculations [16]. In particular, the member of the $0^{+}$isospin triplet state is obtained at an excitation energy of $3.79 \mathrm{MeV}$, close to the experimental value of $3.56 \mathrm{MeV}$. This state is of some interest for the study of the isospin and parity violations 23.

The lowest " $2 \hbar \Omega$ " state that we obtain has $J^{\pi}=$ $1^{+}, T=0$ and an excitation energy of $14.72 \mathrm{MeV}$. It has the configuration of $2 \%|0 \hbar \Omega\rangle+56 \%|2 \hbar \Omega\rangle+22 \%|4 \hbar \Omega\rangle+$ $19 \%|6 \hbar \Omega\rangle$. We identify the second lowest " $2 \hbar \Omega$ " state at $16.08 \mathrm{MeV}$ as the experimental $15.8 \mathrm{MeV}$ state 24] since it has $J^{\pi}=3^{+}$. This state has very little overlap with $0 \hbar \Omega$ configurations; its wave function can be expressed as $58 \%|2 \hbar \Omega\rangle+21 \%|4 \hbar \Omega\rangle+21 \%|6 \hbar \Omega\rangle$.

Below these " $2 \hbar \Omega$ " states we obtain five negativeparity " $1 \hbar \Omega$ " states with excitation energies of 10.9 to 14.2 MeV. One should not be surprised if the experimental energies of these " $1 \hbar \Omega$ " states turn out to be somewhat lower than the values listed in Table IV, obtained in a $5 \hbar \Omega\left(N_{\max }=7\right)$ calculation. We have seen in the cases of ${ }^{4} \mathrm{He}$ and ${ }^{5} \mathrm{He}$ that the excitation energies of the " $1 \hbar \Omega$ " states are lowered by 1 to $3 \mathrm{MeV}$ as we go from a $5 \hbar \Omega$ space to a $7 \hbar \Omega$ space. However we are not able to perform a $7 \hbar \Omega\left(N_{\max }=9\right)$ calculation for the negativeparity states in ${ }^{6} \mathrm{Li}$ at the present time.

A $6 \hbar \Omega$ calculation for ${ }^{6} \mathrm{Li}$ was also attempted by Bevelacqua [25] who used a modified Sussex interaction [13. In that work, all experimentally known states were quite well reproduced. But several " $0 \hbar \Omega$ " and " $1 \hbar \Omega$ " states that we obtain here were not given in [25]. Some of these states, however, were obtained by van Hees et al. 26] in a $(0+1) \hbar \Omega$ calculation using a phenomenological interaction. For example, they obtained a $2^{-}$state at an energy of about $9 \mathrm{MeV}$, lower than our $2^{-}$state at 10.86 $\mathrm{MeV}$.

The g.s. magnetic dipole moment is calculated to be $0.840 \mu_{N}$, slightly larger than the experimental value of $0.822 \mu_{N}$. The g.s. quadrupole moment is calculated to be $-0.067 e \mathrm{fm}^{2}$, very close to the experimental value of $-0.082 e \mathrm{fm}^{2}$. These results are obtained by using bare electromagnetic operators. In principle, these electromagnetic operators should also be renormalized in a way consistent with how the effective interaction is derived from the bare $N N$ potential. This is particularly important when the model space is small. While we hope that our model spaces are large enough to permit the use of bare operators, we are aware that this assumption ought to be verified by explicit calculations of effective operators.

\section{D. ${ }^{7} \mathbf{L i}$}

The negative-parity states are calculated in a full $4 \hbar \Omega$ space $\left(N_{\max }=7\right)$ and the positive-parity states in a full $5 \hbar \Omega$ space $\left(N_{\max }=8\right)$. The results are given in Table $\mathrm{V}$ (see also Fig.5). The theoretical spectrum appears expanded relative to experiment, perhaps indicating that, for the model spaces we can handle, that the two-body $G$ matrix is not an adequate approximation to $V^{\text {eff }}$.

However, the energy of the first excited state $\left(1 / 2^{-}\right)$ agrees very well with experiment $(0.46 \mathrm{MeV}$ vs 0.48 $\mathrm{MeV})$. We had previously experienced some difficulty with this state in single- $G$ calculations using smaller spaces [3], finding excitation energies that were too low. The inclusion of high-lying unperturbed configurations is important for reproducing this state at the experimental energy. In a $0 \hbar \Omega$ calculation, the excitation energy of this state is only $0.195 \mathrm{MeV}$. When $2 \hbar \Omega$ configurations are included, the result increases to $0.498 \mathrm{MeV}$, which becomes $0.463 \mathrm{MeV}$ when $4 \hbar \Omega$ configurations are taken into account.

The lowest positive-parity state we obtain has $J^{\pi}=$ $1 / 2^{+}$and $T=1 / 2$ and an excitation energy of $15.264 \mathrm{MeV}$. This state is dominated by the configurations $(0 s)^{3}(0 p)^{4}$ (about 50\%) $(0 s)^{2}(0 p)^{4}(1 s)^{1}(12 \%)$, and 
$(0 s)^{4}(0 p)^{2}(1 s)^{1}(10 \%)$. The other $28 \%$ is distributed over many configurations.

For the g.s. electric quadrupole moment $Q$, we obtain $-2.37 e \mathrm{fm}^{2}$, much smaller in magnitude than the experimental value of $-4.06 \mathrm{efm}^{2}$. We notice that the calculated quadrupole moment increases in magnitude with the size of the model space. The results for $Q$ from the $0 \hbar \Omega, 2 \hbar \Omega$, and $4 \hbar \Omega$ calculations are $-1.67,-2.16$ and $-2.37 \mathrm{efm}^{2}$, respectively. Presumably still larger model spaces are needed to generate the degree of deformation indicated by the quadrupole moment. However, we are currently unable to go beyond $4 \hbar \Omega$ for this nucleus. One may notice from Table $\mathrm{V}$ that the calculated rms point charge radius is also too small (2.06 fm versus $2.29 \mathrm{fm}$ from experiment), indicating the calculated wave function is probably confined to too small a region by the limited size of the model space. We have repeated the $4 \hbar \Omega$ calculation for $\hbar \Omega=11 \mathrm{MeV}$. The results are also listed in Table V. Although the rms radius for this choice of $\hbar \Omega$ agrees quite well with experiment, the result on the quadrupole moment $Q\left(-2.67 \mathrm{efm}^{2}\right)$, though improved somewhat, is still too small. Therefore changing the model space through adjustments in the basis parameter $\hbar \Omega$ alone is not sufficient, given our use of the bare operator; one has to introduce higher configurations to realistically describe this deformed nucleus.

\section{DEPENDENCE ON THE SIZE OF THE MODEL SPACE}

In this section we examine the differences arising from the use of a multi-valued $G$ matrix, rather than a conventional single-valued effective interaction. These differences are expected to diminish as the model space is increased because the increasing energy denominators in Eq. (7) suppress effects higher order in $\tilde{V}$. In Table VI, the calculated energy and root-mean-square (rms) proton point radius of the ground state and the excitation energy of the first excited state in ${ }^{4} \mathrm{He}$ are given for four different model spaces $\left(N_{\max }=2,4,6\right.$ and 8) and two choices of $\hbar \Omega(14$ and $20 \mathrm{MeV}$ ). As expected (see also Fig.6), the differences between the excitation energies obtained in the conventional and multi-valued $G$-matrix calculations diminish as the model spaces increase. Similarly, the choice of $\hbar \Omega$ becomes less important in the larger model spaces. Note in particular that the calculated g.s. rms radius is about the same $(\sim 1.49 \mathrm{fm})$ in the $8 \hbar \Omega$, multivalued $G$ calculations for the two values of $\hbar \Omega$, indicating good convergence for this quantity.

It is clear from Table VI that the increased size of the model space and the use of an appropriate (multivalued) $G$ matrix both contribute to the improved results for the $0_{2}^{+}$state in ${ }^{4} \mathrm{He}$ in this work. For example, in a conventional (single-valued) $G$-matrix calculation with $\hbar \Omega=14 \mathrm{MeV}$, the excitation energy of this state decreases by $0.55 \mathrm{MeV}$ from $22.93 \mathrm{MeV}$ to 22.38
$\mathrm{MeV}$ when we go from a $6 \hbar \Omega$ space to a $8 \hbar \Omega$ space; in the $8 \hbar \Omega$ space, the use of the multi-valued $G$ matrix further decreases the result by another $0.56 \mathrm{MeV}$ to 21.82 $\mathrm{MeV}$.

As mentioned in Sec. III A, the relative importance of different major-shell configurations depends on the choice of $\hbar \Omega$. For $\hbar \Omega=14 \mathrm{MeV}$, the configurations of the $0_{1}^{+}$ and $0_{2}^{+}$states in ${ }^{4} \mathrm{He}$ obtained in $8 \hbar \Omega$, multi-valued $G$ matrix calculation are given in Eqs. (12,13). For $\hbar \Omega=20$ $\mathrm{MeV}$, we obtain

$$
\begin{aligned}
{\left[0_{1}^{+}\right] \simeq } & 86 \%|0 \hbar \Omega\rangle+4 \%|2 \hbar \Omega\rangle+5 \%|4 \hbar \Omega\rangle \\
& +2 \%|6 \hbar \Omega\rangle+3 \%|8 \hbar \Omega\rangle
\end{aligned}
$$

and

$$
\begin{aligned}
{\left[0_{2}^{+}\right] \simeq } & 0 \%|0 \hbar \Omega\rangle+60 \%|2 \hbar \Omega\rangle+20 \%|4 \hbar \Omega\rangle \\
& +15 \%|6 \hbar \Omega\rangle+5 \%|8 \hbar \Omega\rangle .
\end{aligned}
$$

A comparison of the g.s. configurations in Eqs. (12) and (16) for the two values of $\hbar \Omega$ shows that $\hbar \Omega=20 \mathrm{MeV}$ may be a more reasonable choice for the ground state, since the g.s. can be better approximated as a $0 \hbar \Omega$ state. However, from Eqs. (13) and (17), we can see that the wave function of the first excited state has stronger $6 \hbar \Omega$ and $8 \hbar \Omega$ components (which means slower convergence with respect to the size of the model space) for $\hbar \Omega=20$ $\mathrm{MeV}$ than for $\hbar \Omega=14 \mathrm{MeV}$. This is not surprising, as the $\mathrm{O}_{2}^{+}$state in ${ }^{4} \mathrm{He}$ is loosely bound and has a much larger radius than the $0_{1}^{+}$state. Since it is generally much more difficult to obtain a converged result for the $0_{2}^{+}$state than for the $0_{1}^{+}$state, a basis which leads to faster convergence of the $0_{2}^{+}$state is obviously better when both states are desired. In this sense, $\hbar \Omega=14 \mathrm{MeV}$ is a better choice than $\hbar \Omega=20 \mathrm{MeV}$ for ${ }^{4} \mathrm{He}$.

\section{CONCLUSION}

In a multi- $\hbar \Omega$ model space the two-body $G$ matrix is dependent on the unperturbed energy of the other A2 nucleons. We have used such a multi-valued $G$ matrix in large, no-core, shell-model calculations for light nuclei. When compared to conventional calculations, proper treatment of the $N_{\text {sum }}^{\text {spectators }}$ dependence of the $G$ matrix tends to lower the energies of the " $1 \hbar \Omega$ " and " $2 \hbar \Omega$ " excited states more than the " $0 \hbar \Omega$ " states, bringing energies into better agreement with experiment.

Applying this approach to large, no-core, shell-model calculations, we have achieved a reasonable description of the "low-lying" states (including " $1 \hbar \Omega$ " and " $2 \hbar \Omega$ " states) in light nuclei. With model spaces consisting of as many as nine $\mathrm{HO}$ major shells, the experimentally known states in ${ }^{4} \mathrm{He},{ }^{5} \mathrm{He},{ }^{6} \mathrm{Li}$, and ${ }^{7} \mathrm{Li}$ have been reproduced. Very good agreement with experiment has been obtained for the excited states in ${ }^{4} \mathrm{He}$, the "singleparticle" $3 / 2^{-}-1 / 2^{-}$splitting in ${ }^{5} \mathrm{He}$ and in ${ }^{7} \mathrm{Li}$, and 
the low-lying spectrum of ${ }^{6} \mathrm{Li}$, etc.. Some earlier theoretical predictions of additional states in the spectrum have been confirmed [e.g., a $1 / 2^{+}$state at $4.3 \mathrm{MeV}$ and two nearly degenerate states $\left(3 / 2^{+}\right.$and $\left.5 / 2^{+}\right)$at $9.7 \mathrm{MeV}$ in ${ }^{5} \mathrm{He}$. We have also obtained a few low-lying states that have neither been observed experimentally nor predicted theoretically before. For example, we obtain a $3 / 2^{-}$state at $12.0 \mathrm{MeV}$ in ${ }^{5} \mathrm{He}$ and several " $0 \hbar \Omega$ " and " $1 \hbar \Omega$ " states below $15 \mathrm{MeV}$ in ${ }^{6} \mathrm{Li}$. One shortcoming is that the calculated quadrupole moment of the ground state in ${ }^{7} \mathrm{Li}$ is too small in magnitude when compared with experiment. We attribute this disagreement to the relatively small size $(4 \hbar \Omega)$ of the model space that is used for this nucleus; it may be that the bare quadrupole operator is not appropriate for this space.

By using large, no-core model spaces, we have eliminated adjustable s.p. energies usually involved with shellmodel calculations using effective interactions. However, it should be emphasized that in calculating the $G$ matrices, we have used an empirical prescription for the starting energy, which involves a parameter $\Delta$. This parameter is adjusted to yield a reasonable binding energy. For this reason, our calculated binding energies should not be confused as exact results, which can only be obtained through a parameter-free approach. Recent GFMC calculations of Pudliner et al. 16 serve as a major step in this direction. Nevertheless, we believe that once this parameter is adjusted to reproduce the binding energy, other nuclear properties can then be predicted.

There are important improvements that could be incorporated into future calculations of the type reported here. Our use of very large model spaces was motivated by the hope that bare operators and effective interactions approximated by a two-body $G$ matrix might be successful in such spaces. But presumably the need for large values of $\Delta$ is connected with the omission of the folded diagrams and neglected interactions of three-body and higher clusters in the excluded space. As there are prospects for improving these aspects of the calculations [2], we consider the present effort a first step toward the ultimate goal of accurate shell model calculations based on realistic $N N$ interactions.

If one were able to generate the exact $V^{\text {eff }}$, energy eigenvalues should not depend on the choice of the model space. Thus perhaps the most important result from this initial exploration of multi-valued $G$ matrices is that some improvement was achieved in the rate of convergence of energy eigenvalues, as a function of the complexity of the model space (see, for example, Fig.6). We would argue that the degree to which our results can be further improved is an open question: clearly we have the capacity to put substantial new physics into calculations of $V^{\text {eff }}$ and to generate the corresponding effective operators.

\section{ACKNOWLEDGMENTS}

We thank John Millener and Karlheinz Langanke for useful communications. B.R.B. and D.C.Z. acknowledge partial support of this work by the National Science Foundation, Grant No. PHY93-21668. J.P.V. acknowledges partial support by the U.S. Department of Energy under Grant No. DE-FG02-87ER-40371, Division of High Energy and Nuclear Physics. W.C.H. and C.L.S. acknowledge partial support of this work by the U.S. Department of Energy.

[1] W. C. Haxton and C. Johnson, Phys. Rev. Lett. 651325 (1990).

[2] W.C. Haxton, C.L. Song, J.P. Vary, B.R. Barrett and D.C. Zheng, in preparation.

[3] D.C. Zheng, B.R. Barrett, L. Jaqua, J.P. Vary and R.J. McCarthy, Phys. Rev. C 481083 (1993).

[4] D.C. Zheng, J.P. Vary, and B.R. Barrett, Phys. Rev. C 50, 2841 (1994).

[5] K.A. Bruckner, Phys. Rev. 97, 1353 (1955); 100, 36 (1955).

[6] L. Jaqua, D.C. Zheng, B.R. Barrett, and J.P. Vary, Phys. Rev. C 48, 1765 (1993).

[7] B.R. Barrett, R.G.L. Hewitt, and R.J. McCarthy, Phys. Rev. C 3, 1137 (1971).

[8] V.G.J. Stoks, R.A.M. Klomp, C.P.F. Terheggen, and J.J. de Swart, Phys. Rev. C49, 2950 (1994).

[9] J.P. Vary and D.C. Zheng, "The Many-FermionDynamics Shell-Model Code", Iowa State University (1994) (unpublished).

[10] D.R. Tilley, H.R. Weller, and G.M. Hale, Nucl. Phys. A541, 1 (1992).

[11] I. Tanihata et al., Phys. Rev. Lett. 55, 2676 (1985).

[12] R. Ceuleneer, P. Vandepeutte, and C. Semay, Phys. Lett. B196, 303 (1987); Phys. Rev. C 38, 2335 (1988).

[13] J.P. Elliott, A.D. Jackson, H.A. Mavromatis, E.A. Sanderson, and B. Singh, Nucl. Phys. A121, 241 (1968).

[14] R. A. Arndt and L. D. Roper, Nucl. Phys. A209, 447 (1973).

[15] C.L. Woods, F.C. Barker, W.N. Catford, L.K. Fifield, and N.A. Orr Aust. J. Phys. 41, 525 (1988).

[16] B.S. Pudliner, V.R. Pandharipande, J. Carlson, R.B. Wiringa, "Quantum Monte Carlo Calculations of $A \leq 6$ Nuclei," preprint, (1995).

[17] A.G.M. van Hees and P.W.M. Glaudemans, Z. Phys. A 314, 323(1983); loc. cit., A 315, 223 (1984).

[18] F.C. Barker and C.L. Woods, Aust. J. Phys. 38, 563 (1985).

[19] F. Ajzenberg-Selove, Nucl. Phys. A490, 1 (1988).

[20] A.A. Wolters, A.G.M. van Hees, and P.W.M. Glaudemans, Phys. Rev. C 42, 2053(1990); op. cit., 2062(1990).

[21] D.J. Millener, A.C. Hayes, and D. Strottman, Phys. Rev. C 45, 473 (1992). 
[22] R.C. Barrett and D.F. Jackson, "Nuclear Sizes and Structure" (Clarendon, Oxford, 1977), p.146.

[23] R.G.H. Robertson et al., Phys. Rev. C 29, 755 (1984); R.G.H. Robertson and B.A. Brown, Phys. Rev. C 28, 443 (1983).

[24] B. Jenny, W. Grüebler, V. König, P. Schmelzbach, and C. Schweizer, Nucl. Phys. A397, 61 (1983); B. Jenny, W. Grüebler, V. König, and P. Schmelzbach, Nucl. Phys. A444, 93 (1985).

[25] J.J. Bevelacqua, Nucl. Phys. A357, 126 (1981).

[26] A.G.M. van Hees and P.W.M. Glaudemans, Z. Phys. A 314, 323 (1983); loc. cit., 315, 223 (1984).
TABLE I. Some diagonal matrix elements $\langle(a b$ : $\left.J T)\left|V^{\text {eff }}\right|(a b: J T)\right\rangle$ (in $\mathrm{MeV}$ ) for four possible values of $N_{\text {sum }}$ in a full $6 \hbar \Omega$ calculation of the positive-parity states in ${ }^{6} \mathrm{Li}$.

\begin{tabular}{c|cccc}
\hline \hline$N_{\text {sum }}$ & 2 & 4 & 6 & 8 \\
\hline$(a b: J T)=\left(0 s_{1 / 2} 0 s_{1 / 2}: 01\right)$ & -6.689 & -6.734 & -6.894 & -7.371 \\
$(a b: J T)=\left(0 s_{1 / 2} 0 s_{1 / 2}: 10\right)$ & -8.272 & -9.006 & -9.969 & -11.554 \\
$(a b: J T)=\left(0 s_{1 / 2} 0 p_{3 / 2}: 10\right)$ & -1.144 & -1.415 & -1.769 & -2.344 \\
$(a b: J T)=\left(0 s_{1 / 2} 0 p_{3 / 2}: 11\right)$ & -3.768 & -3.812 & -3.935 & -4.273 \\
$(a b: J T)=\left(0 s_{1 / 2} 0 p_{3 / 2}: 20\right)$ & -8.272 & -9.006 & -9.969 & -11.554 \\
$(a b: J T)=\left(0 s_{1 / 2} 0 p_{3 / 2}: 21\right)$ & -1.006 & -1.029 & -1.058 & -1.090 \\
$(a b: J T)=\left(0 p_{3 / 2} 0 p_{3 / 2}: 01\right)$ & -3.227 & -3.256 & -3.342 & -3.588 \\
$(a b: J T)=\left(0 p_{3 / 2} 0 p_{3 / 2}: 10\right)$ & -1.272 & -1.575 & -1.950 & -2.522 \\
$(a b: J T)=\left(0 p_{3 / 2} 0 p_{3 / 2}: 21\right)$ & -1.364 & -1.389 & -1.439 & -1.545 \\
$(a b: J T)=\left(0 p_{3 / 2} 0 p_{3 / 2}: 30\right)$ & -4.179 & -4.528 & -5.021 & -5.821 \\
\hline \hline
\end{tabular}

Table II. The results for ${ }^{4} \mathrm{He}$ from a full $8 \hbar \Omega\left[N_{\max }=8\right]$ calculation for the positive-parity states and a full $7 \hbar \Omega$ $\left[N_{\max }=7\right]$ calculation for the negative-parity states. In the Table, $E_{B}$ is the binding energy and $E_{x}\left(J_{n}^{\pi}, T\right)$ the excitation energy of the $J_{n}^{\pi}, T$ state. All energies are in $\mathrm{MeV}$. The dominant major-shell configuration for each state is given in the column labeled "Main Conf.". The g.s. rms point radius for protons $\sqrt{\left\langle r_{p}^{2}\right\rangle}$ is also given. The "experimental" g.s. rms radius is deduced from the charge rms radius $\sqrt{\left\langle r_{c}^{2}\right\rangle}$ through (ignoring the neutron charge distribution and other higher-order effects and assuming a proton rms charge radius of $0.81 \mathrm{fm}$ ) $\left\langle r_{p}^{2}\right\rangle=\left\langle r_{c}^{2}\right\rangle-0.81^{2}$.

\begin{tabular}{c|ccc}
\hline \hline Observable & Main Conf. & Mult-valued $G$ & Experiment $^{a)}$ \\
\hline$E_{B}$ & - & 26.459 & 28.296 \\
$\sqrt{\left\langle r_{p}^{2}\right\rangle}(\mathrm{fm})$ & - & 1.492 & 1.46 \\
$E_{x}\left(0_{1}^{+}, 0\right)$ & $0 \hbar \Omega$ & 0 & 0 \\
$E_{x}\left(0_{2}^{+}, 0\right)$ & $2 \hbar \Omega$ & 21.824 & 20.21 \\
$E_{x}\left(0_{1}^{-}, 0\right)$ & $1 \hbar \Omega$ & 21.566 & 21.01 \\
$E_{x}\left(2_{1}^{-}, 0\right)$ & $1 \hbar \Omega$ & 23.003 & 21.84 \\
$E_{x}\left(2_{1}^{-}, 1\right)$ & $1 \hbar \Omega$ & 24.214 & 23.33 \\
$E_{x}\left(1_{1}^{-}, 1\right)$ & $1 \hbar \Omega$ & 24.418 & 23.64 \\
$E_{x}\left(1_{1}^{-}, 0\right)$ & $1 \hbar \Omega$ & 25.286 & 24.25 \\
$E_{x}\left(0_{1}^{-}, 1\right)$ & $1 \hbar \Omega$ & 25.370 & 25.28 \\
$E_{x}\left(1_{2}^{-}, 1\right)$ & $1 \hbar \Omega$ & 25.671 & 25.95 \\
\hline \hline
\end{tabular}

a) From Ref. 10 except for the rms radius which is from Ref. [11]. 
Table III. The results for ${ }^{5} \mathrm{He}$ from a full $6 \hbar \Omega\left[N_{\max }=\right.$ 7] calculation for the negative-parity states and a full $7 \hbar \Omega\left[N_{\max }=8\right]$ calculation for the positive-parity states. Calculated states with an excitation energy larger than $23 \mathrm{MeV}$ are not shown. All the states listed in this table have an isospin $T=1 / 2$. The g.s. electric quadrupole moment $Q$ and magnetic dipole moment $\mu$ are also listed. See the caption of Table II for more explanations.

\begin{tabular}{c|ccc}
\hline \hline Observable & Main Conf. & Multi-valued $G$ & Experiment $^{a)}$ \\
\hline$E_{B}$ & - & 25.883 & 27.410 \\
$\sqrt{\left\langle r_{p}^{2}\right\rangle}(\mathrm{fm})$ & - & 1.630 & $\mathrm{~N} / \mathrm{A}$ \\
$\mu\left(\mu_{N}\right)$ & - & -1.847 & $\mathrm{~N} / \mathrm{A}$ \\
$Q\left(e \mathrm{fm}^{2}\right)$ & - & -0.443 & $\mathrm{~N} / \mathrm{A}$ \\
$E_{x}\left(3 / 2_{1}^{-}\right)$ & $0 \hbar \Omega$ & 0 & 0 \\
$E_{x}\left(1 / 2_{1}^{-}\right)$ & $0 \hbar \Omega$ & 2.465 & $4 \pm 1^{b)}$ \\
$E_{x}\left(1 / 2_{1}^{+}\right)$ & $1 \hbar \Omega$ & 4.343 & see $^{c)}$ \\
$E_{x}\left(3 / 2_{1}^{+}\right)$ & $1 \hbar \Omega$ & 9.717 & see $^{c}$ \\
$E_{x}\left(5 / 2_{1}^{+}\right)$ & $1 \hbar \Omega$ & 9.727 & see \\
$E_{x}\left(3 / 2_{2}^{-}\right)$ & $2 \hbar \Omega$ & 12.006 & $\mathrm{~N} / \mathrm{A}$ \\
$E_{x}\left(1 / 2_{2}^{-}\right)$ & $2 \hbar \Omega$ & 15.213 & $\mathrm{~N} / \mathrm{A}$ \\
$E_{x}\left(7 / 2_{1}^{-}\right)$ & $2 \hbar \Omega$ & 17.252 & $\mathrm{~N} / \mathrm{A}$ \\
$E_{x}\left(5 / 2_{1}^{-}\right)$ & $2 \hbar \Omega$ & 17.296 & $\mathrm{~N} / \mathrm{A}$ \\
$E_{x}\left(3 / 2_{2}^{+}\right)$ & $1 \hbar \Omega$ & 19.060 & 16.75 \\
$E_{x}\left(1 / 2_{2}^{+}\right)$ & $1 \hbar \Omega$ & 19.895 & $\mathrm{~N} / \mathrm{A}$ \\
$E_{x}\left(7 / 2_{1}^{+}\right)$ & $1 \hbar \Omega$ & 21.908 & $\mathrm{~N} / \mathrm{A}$ \\
$E_{x}\left(1 / 2_{3}^{+}\right)$ & $1 \hbar \Omega$ & 22.187 & $\mathrm{~N} / \mathrm{A}$ \\
$E_{x}\left(9 / 2_{1}^{+}\right)$ & $1 \hbar \Omega$ & 22.723 & $\mathrm{~N} / \mathrm{A}$ \\
\hline \hline
\end{tabular}

a) From Ref. [19].

b) Analyses of experiments give different values ranging from $1.4 \mathrm{MeV}$ to $5.5 \mathrm{MeV}$.

c) Previous theoretical works predict a $1 / 2^{+}$state at 5-7 $\mathrm{MeV}$ and a $3 / 2^{+}$and a $5 / 2^{+}$state at $12-14 \mathrm{MeV}$, see Refs. [17, 18].
Table IV. The results for ${ }^{6} \mathrm{Li}$ from a full $6 \hbar \Omega\left[N_{\max }=8\right]$ calculation for the positive-parity states and a full $5 \hbar \Omega$ $\left[N_{\max }=7\right]$ calculation for the negative-parity states. Calculated states with an excitation energy larger than $18 \mathrm{MeV}$ are not shown. See the caption of Table II for more explanations.

\begin{tabular}{c|ccc}
\hline \hline Observable & Main Conf. & Multi-valued $G$ & Experiment $^{a)}$ \\
\hline$E_{B}$ & - & 30.525 & 31.996 \\
$\sqrt{\left\langle r_{p}^{2}\right\rangle}(\mathrm{fm})$ & - & 2.11 & 2.41 \\
$\mu\left(\mu_{N}\right)$ & - & 0.840 & 0.822 \\
$Q\left(e \mathrm{fm}^{2}\right)$ & - & -0.067 & -0.082 \\
$E_{x}\left(1_{1}^{+}, 0\right)$ & $0 \hbar \Omega$ & 0 & 0 \\
$E_{x}\left(3_{1}^{+}, 0\right)$ & $0 \hbar \Omega$ & 2.619 & 2.186 \\
$E_{x}\left(0_{1}^{+}, 1\right)$ & $0 \hbar \Omega$ & 3.786 & 3.563 \\
$E_{x}\left(2_{1}^{+}, 0\right)$ & $0 \hbar \Omega$ & 4.713 & 4.31 \\
$E_{x}\left(2_{1}^{+}, 1\right)$ & $0 \hbar \Omega$ & 6.406 & 5.366 \\
$E_{x}\left(1_{2}^{+}, 0\right)$ & $0 \hbar \Omega$ & 6.764 & 5.65 \\
$E_{x}\left(2_{2}^{+}, 1\right)$ & $0 \hbar \Omega$ & 9.942 & $\mathrm{~N} / \mathrm{A}$ \\
$E_{x}\left(1_{1}^{+}, 1\right)$ & $0 \hbar \Omega$ & 10.742 & $\mathrm{~N} / \mathrm{A}$ \\
$E_{x}\left(2_{1}^{-}, 0\right)$ & $1 \hbar \Omega$ & 10.863 & $\mathrm{~N} / \mathrm{A}$ \\
$E_{x}\left(1_{1}^{-}, 0\right)$ & $1 \hbar \Omega$ & 11.082 & $\mathrm{~N} / \mathrm{A}$ \\
$E_{x}\left(1_{3}^{+}, 0\right)$ & $0 \hbar \Omega$ & 11.382 & $\mathrm{~N} / \mathrm{A}$ \\
$E_{x}\left(0_{2}^{+}, 1\right)$ & $0 \hbar \Omega$ & 12.934 & $\mathrm{~N} / \mathrm{A}$ \\
$E_{x}\left(0_{1}^{-}, 0\right)$ & $1 \hbar \Omega$ & 13.147 & $\mathrm{~N} / \mathrm{A}$ \\
$E_{x}\left(1_{1}^{-}, 1\right)$ & $1 \hbar \Omega$ & 13.706 & $\mathrm{~N} / \mathrm{A}$ \\
$E_{x}\left(2_{1}^{-}, 1\right)$ & $1 \hbar \Omega$ & 14.242 & $\mathrm{~N} / \mathrm{A}$ \\
$E_{x}\left(1_{4}^{+}, 0\right)$ & $2 \hbar \Omega$ & 14.716 & $\mathrm{~N} / \mathrm{A}$ \\
$E_{x}\left(1_{2}^{-}, 0\right)$ & $1 \hbar \Omega$ & 15.422 & $\mathrm{~N} / \mathrm{A}$ \\
$E_{x}\left(3_{2}^{+}, 0\right)$ & $2 \hbar \Omega$ & 16.083 & 15.8 \\
$E_{x}\left(2_{2}^{-}, 0\right)$ & $1 \hbar \Omega$ & 16.950 & $\mathrm{~N} / \mathrm{A}$ \\
$E_{x}\left(0_{1}^{-}, 1\right)$ & $1 \hbar \Omega$ & 17.328 & $\mathrm{~N} / \mathrm{A}$ \\
$E_{x}\left(0_{3}^{+}, 1\right)$ & $2 \hbar \Omega$ & 17.515 & $\mathrm{~N} / \mathrm{A}$ \\
\hline \hline$E_{2}$ & $-\mathrm{R}$, & & \\
\hline
\end{tabular}

a) From Ref. [19] except for the rms radius which is from Ref. 22]. 
Table V. The results for ${ }^{7} \mathrm{Li}$ from a full $4 \hbar \Omega\left[N_{\max }=\right.$ 7] calculation for the negative-parity states and a full $5 \hbar \Omega\left[N_{\max }=8\right]$ calculation for the positive-parity states. Calculated states with an excitation energy larger than $16 \mathrm{MeV}$ are not shown. See the caption of Table II for more explanations. In this table, we also list the results for $\hbar \Omega=11 \mathrm{MeV}$.

\begin{tabular}{c|cccc}
\hline Observable & Main Conf. & $\hbar \Omega=14$ & $\hbar \Omega=11$ & Experiment $^{a)}$ \\
\hline$E_{B}$ & - & 37.533 & 36.141 & 39.244 \\
$\sqrt{\left\langle r_{p}^{2}\right\rangle}(\mathrm{fm})$ & - & 2.062 & 2.233 & 2.29 \\
$\mu\left(\mu_{N}\right)$ & - & 3.027 & 3.014 & 3.2564 \\
$Q\left(e \mathrm{fm}^{2}\right)$ & - & -2.372 & -2.672 & -4.06 \\
$E_{x}\left(3 / 2_{1}^{-}, 1 / 2\right)$ & $0 \hbar \Omega$ & 0 & 0 & 0 \\
$E_{x}\left(1 / 2_{1}^{-}, 1 / 2\right)$ & $0 \hbar \Omega$ & 0.463 & 0.188 & 0.4776 \\
$E_{x}\left(7 / 2_{1}^{-}, 1 / 2\right)$ & $0 \hbar \Omega$ & 5.249 & 5.872 & 4.63 \\
$E_{x}\left(5 / 2_{1}^{-}, 1 / 2\right)$ & $0 \hbar \Omega$ & 7.325 & 7.006 & 6.68 \\
$E_{x}\left(5 / 2_{2}^{-}, 1 / 2\right)$ & $0 \hbar \Omega$ & 8.857 & 8.473 & 7.4595 \\
$E_{x}\left(3 / 2_{2}^{-}, 1 / 2\right)$ & $0 \hbar \Omega$ & 10.749 & 9.178 & 9.85 \\
$E_{x}\left(7 / 2_{2}^{-}, 1 / 2\right)$ & $0 \hbar \Omega$ & 11.402 & 11.012 & 9.67 \\
$E_{x}\left(1 / 2_{2}^{-}, 1 / 2\right)$ & $0 \hbar \Omega$ & 11.608 & 10.165 & $\mathrm{~N} / \mathrm{A}$ \\
$E_{x}\left(5 / 2_{2}^{-}, 1 / 2\right)$ & $0 \hbar \Omega$ & 12.847 & 11.920 & $\mathrm{~N} / \mathrm{A}$ \\
$E_{x}\left(3 / 2_{1}^{-}, 3 / 2\right)$ & $0 \hbar \Omega$ & 12.961 & 12.592 & 11.24 \\
$E_{x}\left(3 / 2_{3}^{-}, 1 / 2\right)$ & $0 \hbar \Omega$ & 13.237 & 11.837 & $\mathrm{~N} / \mathrm{A}$ \\
$E_{x}\left(1 / 2_{3}^{-}, 1 / 2\right)$ & $0 \hbar \Omega$ & 13.704 & 12.419 & $\mathrm{~N} / \mathrm{A}$ \\
$E_{x}\left(1 / 2_{1}^{+}, 1 / 2\right)$ & $1 \hbar \Omega$ & 15.264 & & $\mathrm{~N} / \mathrm{A}$ \\
$E_{x}\left(1 / 2_{1}^{-}, 3 / 2\right)$ & $0 \hbar \Omega$ & 15.780 & & $\mathrm{~N} / \mathrm{A}$ \\
\hline \hline
\end{tabular}

a) From Ref. [19] except for the rms radius which is from Ref. 22].
Table VI. The results for the g.s. energy (in $\mathrm{MeV}$ ), proton rms radius (in $\mathrm{fm}$ ) and the excitation energy (in $\mathrm{MeV}$ ) of the first excited state in ${ }^{4} \mathrm{He}$ obtained in the multivalued $G(\mathrm{~m}-G)$ and single- $G(\mathrm{~s}-G)$ calculations in different model spaces with two choices of $\hbar \Omega$ (14 and 20 $\mathrm{MeV})$. The difference between the s- $G$ and $\mathrm{m}-G$ results is also given.

\begin{tabular}{|c|c|c|c|c|c|}
\hline$\hbar \Omega$ & $N_{\max }$ & Approach & $E\left(0_{1}^{+}\right)$ & $\sqrt{\left\langle r_{p}^{2}\right\rangle}$ & $E_{x}\left(0_{2}^{+}\right)$ \\
\hline \multirow[t]{12}{*}{$\overline{14}$} & \multirow[t]{3}{*}{2} & $\mathrm{~s}-G$ & -23.18 & 1.57 & 26.38 \\
\hline & & $\mathrm{m}-G$ & -23.64 & 1.56 & 25.17 \\
\hline & & diff. & 0.46 & 0.01 & 1.21 \\
\hline & \multirow[t]{3}{*}{4} & $\mathrm{~s}-G$ & -25.23 & 1.57 & 26.73 \\
\hline & & $\mathrm{m}-G$ & -25.95 & 1.56 & 25.78 \\
\hline & & diff. & 0.72 & 0.01 & 0.95 \\
\hline & \multirow[t]{3}{*}{6} & $\mathrm{~s}-G$ & -25.62 & 1.51 & 22.93 \\
\hline & & $\mathrm{m}-G$ & -26.44 & 1.49 & 22.27 \\
\hline & & diff. & 0.82 & 0.02 & 0.66 \\
\hline & \multirow[t]{3}{*}{8} & $\overline{\mathrm{s}-G}$ & -25.62 & 1.51 & 22.38 \\
\hline & & $\mathrm{m}-G$ & -26.46 & 1.49 & 21.82 \\
\hline & & diff. & 0.84 & 0.02 & 0.56 \\
\hline \multirow[t]{13}{*}{20} & \multirow[t]{3}{*}{2} & $\mathrm{~s}-G$ & -25.62 & 1.38 & 33.05 \\
\hline & & $\mathrm{m}-G$ & -25.94 & 1.37 & 30.56 \\
\hline & & diff. & 0.32 & 0.01 & 2.49 \\
\hline & \multirow[t]{3}{*}{4} & $\overline{\mathrm{s}-G}$ & $\begin{array}{l}-26.34 \\
\end{array}$ & 1.46 & 31.84 \\
\hline & & $\mathrm{m}-G$ & -26.84 & 1.45 & 30.23 \\
\hline & & diff. & 0.50 & 0.01 & 1.61 \\
\hline & \multirow[t]{3}{*}{6} & $\mathrm{~s}-G$ & -25.73 & 1.46 & 26.93 \\
\hline & & $\mathrm{m}-G$ & -26.27 & 1.46 & 25.49 \\
\hline & & diff. & 0.54 & 0.00 & 1.44 \\
\hline & \multirow[t]{3}{*}{8} & $\mathrm{~s}-G$ & -25.21 & 1.49 & 24.71 \\
\hline & & $\mathrm{m}-G$ & -25.82 & 1.48 & 23.35 \\
\hline & & diff. & 0.61 & 0.01 & 1.36 \\
\hline & \multicolumn{2}{|c|}{ Experiment } & -28.30 & 1.46 & 20.21 \\
\hline
\end{tabular}




\section{FIGURE CAPTIONS}

\section{Figure 1}

An illustration of the $Q$ operator appropriate for a full $6 \hbar \Omega$ calculation of ${ }^{6} \mathrm{Li}$. The regions interior to the lines are the $Q=0$ regions defined in Eq. (9). The lines correspond to the possible values of $N_{\text {sum }}^{\text {spectators }}$, which range from 0 to $N_{\max }$. The contour for $N_{\text {sum }}^{\text {spectators }}=0$ is given as a solid line. The wings result from the fact that the spectator nucleons are in a unique configuration (closed $0 s$ shell), in this case, forbidding scattering into the $0 s$ shell. The wings make a negligible contribution numerically and can be ignored. The contours for other values of $N_{\text {sum }}^{\text {spectators }}$ are denoted by dashed lines. Note that a single-valued $G$ matrix would employ a single contour and thus neglect much of the physics governing $V^{\text {eff }}$ in a multi- $\hbar \Omega$ space.

\section{Figure 2}

The calculated and experimental low-lying energy spectrum of ${ }^{4} \mathrm{He}$.

\section{Figure 3}

The calculated and experimental low-lying energy spectrum of ${ }^{5} \mathrm{He}$. The first excited state $\left(1 / 2^{-}\right)$is very broad; its experimental excitation energy is not well defined. Refs. 17, 18 also predict a low-lying $1 / 2^{+}$state at $5-7 \mathrm{MeV}$ and $3 / 2^{+}$and $5 / 2^{+}$states at $12-14 \mathrm{MeV}$. We obtain a few " $2 \hbar \Omega$ " (relative to the g.s.) states (e.g., a $3 / 2^{-}$state at $12.88 \mathrm{MeV}$ ) that have not been observed experimentally nor predicted theoretically before. All the states shown in this Figure have an isospin $T=1 / 2$.

\section{Figure 4}

The calculated and experimental low-lying energy spectrum of ${ }^{6} \mathrm{Li}$.

\section{Figure 5}

The calculated and experimental low-lying energy spectrum of ${ }^{7} \mathrm{Li}$. All the states shown in this figure have an isospin $T=1 / 2$ except for the $3 / 2^{-}$state at 12.96 $\mathrm{MeV}$ which has $T=3 / 2$.
Figure 6

(a) The rms point charge radius of the ground state in ${ }^{4}$ He obtained in multi-valued $G(\mathrm{mG})$ calculations with model spaces of different sizes [signified by $N_{\max }$ ] using two values of the HO basis parameter $\hbar \Omega[14 \mathrm{MeV}$ (solid lines) and $20 \mathrm{MeV}$ (dashed lines)].

(b) Similar to (a) but for the excitation energy of the first excited $0^{+}$state in ${ }^{4} \mathrm{He}$. Results from both the multivalued $G(\mathrm{mG})$ and single- $G(\mathrm{sG})$ calculations are shown. 